\title{
Blowups with log canonical singularities
}

\author{
G.K. Sankaran* Francisco Santos ${ }^{\dagger}$
}

February 25, 2020

\begin{abstract}
We show that the minimum weight of a weighted blow-up of $\mathbb{A}^{d}$ with $\varepsilon$-log canonical singularities is bounded by a constant depending only on $\varepsilon$ and $d$. This was conjectured by Birkar.

Using the recent classification of 4-dimensional empty simplices by Iglesias-Valiño and Santos, we work out an explicit bound for blowups of $\mathbb{A}^{4}$ with terminal singularities: the smallest weight is always at most 32 , and at most 6 in all but finitely many cases.
\end{abstract}

\section{Introduction}

At a meeting of the COW seminar at City, University of London on 7th February 2018, Caucher Birkar asked the following question.

Question 1.1. Denote by $\mathbb{A}_{\mathbf{n}}^{4}$ the weighted blowup of $\mathbb{A}^{4}$ at $0 \in \mathbb{A}^{4}$ with coprime weights $\mathbf{n}=\left(n_{1}, n_{2}, n_{3}, n_{4}\right) \in \mathbb{N}^{4}$. If $\mathbb{A}_{\mathbf{n}}^{4}$ has terminal singularities, is the smallest of the weights bounded?

By "coprime" we mean only that $\mathbf{n}$ is primitive: we do not require the weights to be pairwise coprime.

This is a simplified version of a more ambitious conjecture.

Conjecture 1.2 (Birkar). Denote by $\mathbb{A}_{\mathbf{n}}^{d}$ the weighted blowup of $\mathbb{A}^{d}$ at $0 \in \mathbb{A}^{d}$ with coprime weights $\mathbf{n}=\left(n_{1}, \ldots, n_{d}\right) \in \mathbb{N}^{d}$. If $\mathbb{A}_{\mathbf{n}}^{d}$ has $\varepsilon$-log canonical singularities, then the smallest of the weights is bounded by a constant depending only on $d$ and $\varepsilon$.

Our main result, Theorem 1.3, is a proof of Conjecture 1.2.

${ }^{*}$ Department of Mathematical Sciences, University of Bath, Bath BA2 7AY, UK, G.K.Sankaran@bath.ac.uk

${ }^{\dagger}$ Departamento de Matemáticas, Estadística y Computación, Universidad de Cantabria, 39005 Santander, Spain, francisco.santos@unican.es. Supported by grant MTM2017-83750-P of the Spanish Ministry of Economy and Competitiveness and by the Einstein Foundation Berlin under grant EVF-2015-230 
Theorem 1.3. In each fixed dimension $d$ and for each $\varepsilon \in(0,1]$ there is an integer $\ell_{\varepsilon, d} \in \mathbb{N}$ such that if $\mathbf{n}=\left(n_{1}, \ldots, n_{d}\right) \in \mathbb{N}^{d}$ is primitive and the weighted blowup $\mathbb{A}_{\mathbf{n}}^{d}$ has only $\varepsilon$-log canonical singularities then $n_{\min }:=$ $\min \left\{n_{1}, \ldots, n_{d}\right\} \leq \ell_{\varepsilon, d}$.

Our proof relies on a general result about subgroups of $\mathbb{R}^{n}$ that miss a given open set, due to Lawrence [11], which we state here as Theorem [3.1. The connection of that result to terminal and canonical singularities, and to hollow and empty simplices, was first noticed by A. Borisov [6]. Independently of us, and by somewhat different methods, Y. Chen [7] has proved Conjecture 1.2 for the case $d=3$.

We also give a precise answer to Question 1.1.

Theorem 1.4. If the weighted blowup $\mathbb{A}_{\mathbf{n}}^{4}$ has terminal singularities then $n_{\min } \leq 32$. Moreover, with finitely many exceptions $n_{\min } \leq 6$.

The proof of this statement relies on the complete classification of empty simplices in dimension four due to Iglesias-Valiño and Santos [9]. The bound of 6 is attained by the infinite family of blowups with $\mathbf{n}=(6,10,15, n)$, which have terminal singularities whenever $n$ is coprime with 30 (see Remark4.10). The bound of 32 is attained only by the blowup with $\mathbf{n}=(32,41,71,102)$. There are a total of 1784 blowups of $\mathbb{A}^{4}$ with $n_{\min }>6$; the number of them for each value of $n_{\min }$ is listed in Proposition 4.11.

These results extend a theorem of Kawakita [10, Theorem 3.5], which says that a weighted blowup $\mathbb{A}_{\mathbf{n}}^{3}$ is terminal if and only if the weights are $(1, a, b)$ with $a$ and $b$ coprime. Kawakita's result also follows from our methods: see Corollary 4.4 below.

The context of [10] is the Sarkisov program, in particular birational rigidity. To investigate Sarkisov links involving a Fano 3-fold $F$ of Picard rank 1 requires in principle an understanding of all possible divisorial contractions in the Mori program with target $F$. The main outcome of [10] is that any divisorial contraction in the Mori program with centre a smooth point is a weighted blowup, and [10, Theorem 3.5] says that the weights must then be $(1, a, b)$.

This is important because, at least in dimension 3, we understand divisorial contractions well if we know their sources, but not so well if we know their targets. So [10] provides a description of all possible baskets of singularities in a terminal 3 -fold with a divisorial contraction whose centre is a smooth point. This may be thought of as a relative boundedness result, showing that exceptional divisors are weighted projective planes of the form $\mathbb{P}(1, a, b)$.

Birkar's Conjecture 1.2 arises analogously in his work [3] on boundedness of $\log$ Calabi-Yau fibrations. One way to view it is as a local version of the $\mathrm{BAB}$ conjecture, in a quite special case. 
Acknowledgements: Some background on birational geometry was supplied to GKS by Anne-Sophie Kaloghiros. The explanations here relating these results to their wider context are largely hers, but errors and omissions in such explanations are definitely ours. Parts of this work were carried out while GKS was visiting Fukuoka University and KIAS, Seoul: he thanks both for hospitality and a helpful environment. We also thank the organisers of MEGA 2019 (Madrid), where the two authors first met and discussed these questions.

\section{Singularities and simplices}

Geometrically, our approach is to use toric geometry to rephrase the problem in terms of polytopes. We shall be working in $\mathbb{R}^{d}$ with its standard basis $\mathbf{e}_{1}=(1,0, \ldots, 0), \ldots, \mathbf{e}_{d}$. We shall frequently need to add up the coordinates of a vector, so we write $\Sigma x_{i}$ to abbreviate $\sum_{i=1}^{d} x_{i}$.

Definition 2.1. Let $\Lambda \subseteq \mathbb{R}^{d}$ be a lattice: that is, a finitely generated free abelian subgroup of rank $d$ such that $\mathbb{R}^{d}=\Lambda \otimes \mathbb{R}$. A polytope $\Pi$ in $\mathbb{R}^{d}$ is a bounded intersection of finitely many closed half-spaces. A point $\mathbf{v} \in \Pi$ is a vertex if $\Pi \cap H=\{\mathbf{v}\}$ for some affine hyperplane $H \subset \mathbb{R}^{d}$ : we denote the set of vertices of $\Pi$ by $\operatorname{Vx}(\Pi)$. The convex hull of a set $X \subset \mathbb{R}^{d}$ is denoted $\operatorname{Conv}(X)$ : a polytope $\Pi$ is always equal to the convex hull $\operatorname{Conv}(\operatorname{Vx}(\Pi))$ of its vertices. $\Pi$ is a lattice polytope if $\operatorname{Vx}(\Pi) \subset \Lambda$.

The next definition is usually made only for the case where $\Gamma$ is a lattice and $\Pi$ is a lattice polytope, but we need it in a more general setting.

Definition 2.2. Fix a subgroup $\Gamma$ of $\mathbb{R}^{d}$. We say that a polytope $\Pi$ is hollow with respect to $\Gamma$ if $\Pi \cap \Gamma \subseteq \partial \Pi$ and empty with respect to $\Gamma$ if $\Pi \cap \Gamma \subseteq \operatorname{Vx}(\Pi)$. We omit "with respect to $\Gamma$ " when $\Gamma$ is understood.

Let $\sigma=\sum \mathbb{R}_{\geq 0} \mathbf{w}_{r}$ be a nondegenerate closed rational polyhedral cone in $\mathbb{R}^{d}$, where $\mathbf{w}_{r} \in \Lambda$ are primitive generators of the rays of $\sigma$. We denote by $\Delta(\sigma)$ the lattice polytope $\operatorname{Conv}\left(\{0\} \cup\left\{\mathbf{w}_{i}\right\}\right)$, and let $X_{\sigma}$ be the affine variety Spec $\mathbb{C}\left[\sigma^{\vee} \cap \Lambda\right]$, as usual in toric geometry. With this notation, $X_{\sigma}$ is $\mathbb{Q}$-Gorenstein if and only if all the $\mathbf{w}_{i}$ lie in an affine hyperplane, and is $\mathbb{Q}$-factorial if and only if $\sigma$ is simplicial; that is, if $\Delta(\sigma)$ is a simplex.

The following fundamental fact is well known.

Lemma 2.3. Let $\varepsilon \in(0,1]$. Then:

(a) $X_{\sigma}$ is $\varepsilon$-log terminal if and only if $\varepsilon \Delta(\sigma)$ is an empty polytope.

(b) $X_{\sigma}$ is $\varepsilon$-log canonical if and only if $\varepsilon \Delta(\sigma)$ is hollow and all nonzero lattice points in it lie in facets not containing the origin. 
Proof. $X_{\sigma}$ is $\varepsilon-\log$ canonical if and only if for some (hence any) birational morphism $f: Y \rightarrow X_{\sigma}$ with $Y$ smooth, the discrepancies $e_{j}$ defined by $K_{Y}-$ $f^{*} K_{X}=\sum_{j} e_{j} E_{j}$ (with $E_{j}$ being $f$-exceptional prime divisors) satisfy $e_{j} \geq$ $-1+\varepsilon$. To check this, consider a toric resolution $f: Y=Y_{\Sigma} \rightarrow X_{\sigma}$ obtained by subdividing $\sigma$ into a regular fan $\Sigma$. The exceptional divisors are given by some rays $\rho_{j}$ spanned by primitive $\mathbf{r}_{j} \in \Lambda$. The $\mathbb{Q}$-divisors $K_{Y}$ and $f^{*} K_{X_{\sigma}}$ are given by support functions $h_{Y}$ and $h_{X_{\sigma}}$ as in [15, Proposition 2.1(v)]. The function $h_{Y}$ satisfies $h_{Y}\left(\mathbf{r}_{j}\right)=h_{Y}\left(\mathbf{w}_{i}\right)=1$, while $h_{X_{\sigma}}$ is linear and is determined by $h_{X_{\sigma}}\left(\mathbf{w}_{i}\right)=0$. Therefore $e_{j}=-1+h_{X_{\sigma}}\left(\mathbf{r}_{j}\right)$, so in part (b) we have $h_{X_{\sigma}}(\mathbf{r}) \geq \varepsilon$, for all $\mathbf{r} \in \Lambda$. The result follows at once from this: part (a) is identical, replacing $e_{j} \geq-1+\varepsilon$ by $e_{j}>-1+\varepsilon$.

In particular, since canonical is the same as $1-\log$ canonical, $X_{\sigma}$ has $\mathbb{Q}$ factorial canonical singularities if and only if $\Delta(\sigma)$ is a hollow simplex with $\Delta(\sigma) \cap \Lambda \backslash\{0\}$ contained in the facet opposite to the origin.

Any nonnegative primitive integer vector $\mathbf{n}=\left(n_{1}, \ldots, n_{d}\right) \in \mathbb{N}^{d}$ induces a weighted blowup $\mathbb{A}_{\mathbf{n}}^{d}$, which is the toric variety associated with the fan in $\mathbb{R}^{d}$ (and the lattice $\mathbb{Z}^{d}$ ) that consists of all the faces of the cones $\sigma_{\mathbf{n}}^{j}=$ $\mathbb{R}_{\geq 0} \mathbf{n}+\sum_{i \neq j} \mathbb{R}_{\geq 0} \mathbf{e}_{i}$. Note that all such faces are contained in $\mathbb{R}_{\geq 0}^{d}$, and that the $\sigma_{\mathbf{n}}^{j}$ are simplicial so $\mathbb{A}_{\mathbf{n}}^{d}$ always has $\mathbb{Q}$-factorial singularities.

The standard simplex in $\mathbb{R}^{d}$ is $\Delta:=\Delta\left(\mathbb{R}_{\geq 0}^{d}\right)=\operatorname{Conv}\left(\left\{0, \mathbf{e}_{1}, \ldots, \mathbf{e}_{d}\right\}\right)$ and its interior is denoted $\Delta^{\circ}$. That is,

$$
\Delta^{\circ}=\left\{\mathbf{x} \in \mathbb{R}^{d} \mid \Sigma x_{i}<1 \text { and } \forall i x_{i}>0\right\} .
$$

The facet of $\Delta$ opposite to the origin, which is $\operatorname{Conv}\left(\left\{\mathbf{e}_{1}, \ldots, \mathbf{e}_{d}\right\}\right)$, is denoted by $\Delta_{1}$.

For any non-zero $\mathbf{n} \in \mathbb{N}^{d}$ we set $\Delta_{\mathbf{n}}=\operatorname{Conv}\left(\left\{\mathbf{e}_{1}, \ldots, \mathbf{e}_{d}, \mathbf{n}\right\}\right)$.

Proposition 2.4. For $\varepsilon \in(0,1]$

(a) $\mathbb{A}_{\mathbf{n}}^{d}$ has $\varepsilon$-log terminal singularities if and only if $\varepsilon \Delta_{\mathbf{n}}$ is empty.

(b) $\mathbb{A}_{\mathbf{n}}^{d}$ has $\varepsilon$-log canonical singularities if and only if $\varepsilon \Delta_{\mathbf{n}}$ is hollow.

Proof. (a) The singularities of $\mathbb{A}_{\mathbf{n}}^{d}$ are $\varepsilon-\log$ terminal if and only if all the polytopes $\varepsilon \Delta\left(\sigma_{\mathbf{n}}^{j}\right)$ are empty: that is, if $\bigcup_{j=1}^{d} \varepsilon \Delta\left(\sigma_{\mathbf{n}}^{j}\right)$ is empty. But

$$
\begin{aligned}
\bigcup_{i=1}^{n} \varepsilon \Delta_{\sigma_{\mathbf{n}}^{i}} & =\varepsilon \operatorname{Conv}\left(0, \mathbf{e}_{1}, \ldots, \mathbf{e}_{d}, \mathbf{n}\right) \\
& =\varepsilon \operatorname{Conv}\left(0, \mathbf{e}_{1}, \ldots, \mathbf{e}_{d}\right) \cup \varepsilon \operatorname{Conv}\left(\mathbf{e}_{1}, \ldots, \mathbf{e}_{d}, \mathbf{n}\right) \\
& =\varepsilon \Delta \cup \varepsilon \Delta_{\mathbf{n}}
\end{aligned}
$$

and $\varepsilon \operatorname{Conv}\left(\left\{0, \mathbf{e}_{1}, \ldots, \mathbf{e}_{d}\right\}\right)$ is empty anyway. 
(b) All lattice points of $\bigcup_{i=1}^{n} \varepsilon \Delta\left(\sigma_{\mathbf{n}}^{i}\right)$ other than the origin lie in $\varepsilon \Delta_{\mathbf{n}}$ by construction. Hence they all lie in facets not containing the origin if and only if they do not lie in the interior of $\varepsilon \Delta_{\mathbf{n}}$ or in $\varepsilon \Delta_{\mathbf{n}} \cap \varepsilon \Delta=$ $\varepsilon \operatorname{Conv}\left(\left\{\mathbf{e}_{1}, \ldots, \mathbf{e}_{d}\right\}\right)=\varepsilon \Delta_{1}$. The latter is empty, and except for the trivial case $\varepsilon=1$ has no lattice points among its vertices either.

The following change of coordinates sends the simplex $\Delta_{\mathbf{n}}$ of Proposition 2.4 to the standard simplex $\Delta$, which will be useful for us.

Lemma 2.5. Let $\mathbf{n}=\left(n_{1}, \ldots, n_{d}\right) \in \mathbb{R}_{\geq 0}^{d}$ be a non-negative vector with $\Sigma n_{i}>1$. Then the unique affine-linear transformation sending $\mathbf{n}$ to the origin and fixing all of $\mathbf{e}_{1}, \ldots, \mathbf{e}_{d}$ sends the origin to $\mathbf{n} /\left(-1+\Sigma n_{i}\right)$.

Proof. The unique (modulo multiplication by a scalar) affine dependences among $\left\{0, \mathbf{e}_{1}, \ldots, \mathbf{e}_{d}, \mathbf{n}\right\}$ and among $\left\{\mathbf{n} /\left(-1+\Sigma n_{i}\right), \mathbf{e}_{1}, \ldots, \mathbf{e}_{d}, 0\right\}$ are the same one: its coefficients are $\left(1-\Sigma n_{i}, n_{1}, \ldots, n_{d},-1\right)$.

Corollary 2.6. Let $\mathbf{n} \in \mathbb{N}^{d}$. Define $V=-1+\Sigma n_{i}$ and $\mathbf{p}=\frac{1}{V} \mathbf{n} \in \mathbb{Q}^{d}$. Let $\Lambda_{\mathbf{p}}=\mathbb{Z}^{d}+\mathbb{Z} \mathbf{p}$ be the lattice generated by $\mathbf{p}$ and $\mathbb{Z}^{d}$. Then, for any $\varepsilon \in(0,1]$ :

(a) $\mathbb{A}_{\mathbf{n}}^{d}$ has $\varepsilon$-log terminal singularities if and only if $\Delta_{\mathbf{p}, \varepsilon}=\mathbf{p}+\varepsilon(\Delta-\mathbf{p})$ is empty with respect to the lattice $\Lambda_{\mathbf{p}}$.

(b) $\mathbb{A}_{\mathbf{n}}^{d}$ has $\varepsilon$-log canonical singularities if and only if $\Delta_{\mathbf{p}, \varepsilon}$ is hollow with respect to the lattice $\Lambda_{\mathbf{p}}$.

Proof. This is just Proposition 2.4, rephrased via the change of coordinates of Lemma 2.5. The notation here will be used more widely: see Definition 3.2 below.

\section{$3 \quad \varepsilon-\log$ canonical singularities}

This section is devoted to the proof of Theorem 1.3 .

\subsection{Lawrence's Theorem and hollow points}

Apart from the relation between $\varepsilon$-log canonical singularities and hollow simplices described in Corollary 2.6, our main technical tool is the following result of Jim Lawrence (see also [6]).

Theorem 3.1 (Lawrence [11, Theorem 1]). Fix $d \in \mathbb{N}$ and an open subset $U \subset \mathbb{R}^{d}$, and let $\mathbb{G}$ be a closed subgroup of $\mathbb{R}^{d}$ containing $\mathbb{Z}^{d}$. Then there are only finitely many maximal subgroups $G<\mathbb{G}$ such that $\mathbb{Z}^{d} \subset G$ and $G \cap U=\varnothing$. 
In other words, any subgroup of $\mathbb{G}$ that contains $\mathbb{Z}^{d}$ and misses $U$ is contained in one (at least) of finitely many such subgroups of $\mathbb{G}$.

These maximal subgroups $G$ are automatically closed. Hence $G$ is a Lie subgroup of $\mathbb{R}^{d}$, and its identity component, which we call $L$, is a linear subspace of dimension equal to $\operatorname{dim} G$. Some of the groups containing $\mathbb{Z}^{d}$ that we consider below are not closed, however.

The relation to our problem comes from the fact that the lattice $\Lambda_{\mathbf{p}}$ in Corollary 2.6 is a subgroup of $\mathbb{R}^{d}$ containing $\mathbb{Z}^{d}$. This implies, for example, that taking $U=\Delta^{\circ}$, we may interpret the case $\varepsilon=1$ of Corollary [2.6(b) as saying that if $\mathbb{A}_{\mathbf{n}}^{d}$ has only canonical singularities then $\mathbf{p}$ lies in one of finitely many subgroups of $\mathbb{R}^{d}$ containing $\mathbb{Z}^{d}$ and not intersecting $\Delta^{\circ}$.

Our aim is to extend this approach to any value of $\varepsilon \in(0,1]$. We first extend the notation introduced in Corollary 2.6, using Definition 2.2.

Definition 3.2. We define

$$
\Omega:=\mathbb{R}_{\geq 0}^{d} \backslash \Delta=\left\{\mathbf{x} \in \mathbb{R}^{d} \mid \Sigma x_{i}>1 \text { and } \forall i x_{i} \geq 0\right\} .
$$

For each point $\mathbf{p} \in \Omega$ :

(a) We call the number $V:=\frac{1}{-1+\Sigma p_{i}} \in \mathbb{R}_{\geq 0}$ the index of $\mathbf{p}$. The entries of the vector $\mathbf{n}:=V \mathbf{p} \in \mathbb{R}_{>0}^{d}$ are called the weights of $\mathbf{p}$, and the smallest of them is called the smallest weight $n_{\min }=n_{\min }(\mathbf{p})$ of $\mathbf{p}$.

(b) We put $\Delta_{\mathbf{p}, \varepsilon}=\mathbf{p}+\varepsilon(\Delta-\mathbf{p})$ and $\Lambda_{\mathbf{p}}=\mathbb{Z}^{d}+\mathbb{Z} \mathbf{p}$.

(c) We say that $\mathbf{p}$ is $\varepsilon$-hollow if $\Delta_{\mathbf{p}, \varepsilon}$ is hollow with respect to the group $\Lambda_{\mathbf{p}}$.

The notation in Definition 3.2(a) is compatible with the notation of Corollary 2.6 because

$$
-1+\Sigma n_{i}=-1+V \Sigma p_{i}=-1+V\left(\frac{1}{V}+1\right)=V,
$$

but at this stage we do not require the weights to be integers: $V$ and $\mathbf{n}$ need not even be rational, so the group $\Lambda_{\mathbf{p}}$ may not be a lattice.

Observe that $\Delta_{\mathbf{p}, \varepsilon}$ is $\Delta$ shrunk towards $\mathbf{p}$ by a factor $\varepsilon$, so it is a simplex with facets parallel to the facets of $\Delta$.

\subsection{The canonical case of Birkar's conjecture}

We let $H_{0}=\left\{\mathbf{x} \mid \Sigma x_{i}=0\right\}$ and $H_{1}=\left\{\mathbf{x} \mid \Sigma x_{i}=1\right\}$. Thus $H_{1}$ is the affine hyperplane containing $\Delta_{1}$ and $H_{0}$ is the linear hyperplane parallel to it. Let $\Delta_{1}^{\circ}$ denote the relative interior of $\Delta_{1}$.

Fix a linear subspace $L \subset \mathbb{R}^{d}$, of codimension $k$. Assuming that $L \nsubseteq H_{0}$ we are going to prove a bound $\ell_{L}$, depending only on $L$, for the minimum weight of every point $\mathbf{p} \in \Omega$ such that $L+\mathbf{p}$ does not meet $\Delta_{1}^{\circ}$. 
For this, let $\pi_{L}: \mathbb{R}^{d} \rightarrow \mathbb{R}^{d} / L \cong \mathbb{R}^{k}$ be the canonical projection along $L$, let $\mathbf{s}_{i}=\pi_{L}\left(\mathbf{e}_{i}\right)$, and let $S=\left\{0, \mathbf{s}_{1}, \ldots, \mathbf{s}_{d}\right\}$, so that $\operatorname{Conv}(S)=\pi_{L}(\Delta)$. The condition $L \nsubseteq H_{1}$ implies that no affine hyperplane in $\mathbb{R}^{d} / L$, in particular no facet of $\operatorname{Conv}(S)$, contains $\left\{\mathbf{s}_{1}, \ldots, \mathbf{s}_{d}\right\}$. This makes the minimum in the following statement well-defined.

Proposition 3.3. Suppose that $L \subseteq \mathbb{R}^{d}$ is a linear subspace not contained in $H_{1}$. For each facet-supporting hyperplane $H$ of $\pi_{L}(\Delta)$ let

$$
\ell_{H}:=\min _{\mathbf{s}_{i} \notin H} \frac{\operatorname{dist}(H, 0)}{\operatorname{dist}\left(H, \mathbf{s}_{i}\right)},
$$

and let $\ell_{L}=\max _{H} \ell_{H}$. Then every point $\mathbf{p} \in \Omega$ such that $\mathbf{p}+L$ does not meet $\Delta_{1}^{\circ}$ has $n_{\min }(\mathbf{p}) \leq \ell_{L}$.

Remark 3.4. Let $k=d-\operatorname{dim} L$. In $\mathbb{R}^{d} / L \cong \mathbb{R}^{k}$, an affine hyperplane $H$ is expressed as $H=\left\{\mathbf{x} \in \mathbb{R}^{k} \mid f(\mathbf{x})=c\right\}$, where $f: \mathbb{R}^{k} \rightarrow \mathbb{R}$ is a linear functional. For $\mathbf{y} \in \mathbb{R}^{k}$, we define the distance $\operatorname{dist}(H, \mathbf{y})=|f(\mathbf{y})-c|$. This depends on the choice of $f$, which is only unique up to a scalar and, implicitly, on the choice of isomorphism $\mathbb{R}^{d} / L \cong \mathbb{R}^{k}$. But in the statement of Proposition 3.3 and the rest of this section we only consider ratios of two distances, which do not depend on choice. In Section 4 we shall need to be more definite.

Proof. Since $(\mathbf{p}+L) \cap \Delta_{1}^{\circ}=\varnothing$ and $\mathbf{p} \in \Omega$, we also have $(\mathbf{p}+L) \cap \Delta^{\circ}=\varnothing$, and the point $\pi_{L}(\mathbf{p})$ is not in the interior of $\operatorname{Conv}(S)$. Hence there is a facetsupporting hyperplane $H$ of $\operatorname{Conv}(S)$ that weakly separates $\pi_{L}(\mathbf{p})$ from $\operatorname{Conv}(S)$. Let $\widetilde{H}=\pi_{L}^{-1}(H)$, which is a hyperplane weakly separating $L+\mathbf{p}$ from $\Delta$ (but is not necessarily facet-supporting for $\Delta$ ).

If $0 \in \widetilde{H}$ then, in order for $\mathbf{p}$ to be in $\Omega$, one of the coordinates of $\mathbf{p}$, hence one of the weights of $\mathbf{p}$, must be zero. Thus we assume $0 \notin \widetilde{H}$ and we can find an $\mathbf{a} \in \mathbb{R}^{d}$ such that $\widetilde{H}=\left\{\mathbf{x} \in \mathbb{R}^{d} \mid \mathbf{a} \cdot \mathbf{x}=1\right\}$, where $\mathbf{a} \cdot \mathbf{x}:=\sum_{i=1}^{d} a_{i} x_{i}$ is the usual Euclidean inner product.

Since $\widetilde{H}$ weakly separates $\Delta$ from $\mathbf{p}$ we have $\sum_{i} a_{i} p_{i}=\mathbf{a} \cdot \mathbf{p} \geq 1$ but $\mathbf{a} . \mathbf{x} \leq 1$ for every $\mathbf{x} \in \Delta$; in particular, $a_{i}=\mathbf{a} \cdot \mathbf{e}_{i} \leq 1$ for every $i$. Thus

$$
\sum_{i=1}^{d}\left(1-a_{i}\right) n_{i}=\sum_{i=1}^{d} n_{i}-V \sum_{i=1}^{d} a_{i} p_{i} \leq(V+1)-V=1 .
$$

Since the terms in the first sum are non-negative, $\left(1-a_{i}\right) n_{i} \leq 1$ for every $i$.

Observe that $\operatorname{dist}(\widetilde{H}, 0)=1$ and $\operatorname{dist}\left(\widetilde{H}, \mathbf{e}_{i}\right)=\left(1-\mathbf{a . e}_{i}\right)$ so

$$
\frac{\operatorname{dist}\left(H, \mathbf{s}_{i}\right)}{\operatorname{dist}(H, 0)}=\frac{\operatorname{dist}\left(\widetilde{H}, \mathbf{e}_{i}\right)}{\operatorname{dist}(\widetilde{H}, 0)}=1-a_{i}
$$


Hence, for any $i$ with $\mathbf{s}_{i} \notin H$ (which exists, because otherwise we would have $\widetilde{H}=\left\{\Sigma x_{i}=1\right\}=H_{1}$ and that would imply $L \subset H_{0}$ ) we have

$$
n_{i} \leq \frac{1}{1-a_{i}}=\frac{\operatorname{dist}(H, 0)}{\operatorname{dist}\left(H, \mathbf{s}_{i}\right)} .
$$

Thus $n_{\min }(\mathbf{p}) \leq \ell_{H}$. This does not yet give a bound for $n_{\min }(\mathbf{p})$ because $H$ depends on $\mathbf{p}$, but $H$ is one of the finitely many facet-supporting hyperplanes of $\pi_{L}(\Delta)$, so $n_{\min }(\mathbf{p}) \leq \max _{H} \ell_{H}=\ell_{L}$ as claimed.

Although we give below a separate proof of the general case, it is interesting to observe that Proposition 3.3 leads to the following easy proof of the canonical case of Theorem 1.3 .

Proof of Theorem 1.3 for $\varepsilon=1$. By Theorem 3.1 there is a finite collection $\left\{G_{1}, \ldots, G_{t}\right\}$ of closed subgroups of $\mathbb{R}^{d}$ containing $\mathbb{Z}^{d}$ and not meeting $\Delta^{\circ}$, such that any subgroup of $\mathbb{R}^{d}$ containing $\mathbb{Z}^{d}$ and not meeting $\Delta^{\circ}$ is contained in one of them. We denote $L_{j}$ the identity component of $G_{j}$.

If $L_{j} \subset H_{0}$, then the quotient $G_{j} /\left(G_{j} \cap H_{0}\right) \cong \pi_{H_{0}}\left(G_{j}\right)$ is a discrete subgroup of $\mathbb{R}^{d} / H_{0} \cong \mathbb{R}$. Let $y$ be the minimum of $\pi_{H_{0}}\left(G_{j}\right)$ in the interval $(1, \infty)$ and define $\ell_{G_{j}}=1 /(-1+y)$. Then the index (and hence each weight) of every $\mathbf{p} \in G_{j} \cap \Omega$ is bounded by $\ell_{G_{j}}$.

If $L_{j} \not \subset H_{0}$, then Proposition 3.3 applies, since $L_{j}+\mathbf{p} \subset G_{j}$ does not meet $\Delta^{\circ}$. The proposition gives us an $\ell_{G_{j}}=\ell_{L_{j}}$ (depending only on $L_{j}$ ) with $n_{\min }(\mathbf{p}) \leq \ell_{G_{j}}$ for every $\mathbf{p} \in G_{j} \cap \Omega$.

We can then take $\ell_{1, d}=\max _{j=1, \ldots, t} \ell_{G_{j}}$. Indeed, let $\mathbf{n} \in \mathbb{N}^{d}$ be such that $\mathbb{A}_{\mathbf{n}}^{d}$ has only canonical singularities. As above, let $V=-1+\Sigma n_{i}$ and let $\mathbf{p}=\frac{1}{V} \mathbf{n}$, which lies in $\Omega$. By Corollary 2.6 the lattice $\Lambda_{\mathbf{p}}=\mathbb{Z}^{d}+\mathbb{Z} \mathbf{p}$ does not meet $\Delta^{\circ}$ and is thus contained in some $G_{j}$ from our list. Thus, $n_{\min }=n_{\min }(\mathbf{p}) \leq \ell_{G_{j}} \leq \ell_{1, d}$.

\subsection{Local weight bound}

In this section we examine the situation near a given point $\mathbf{x}$ of $\Delta_{1}$ and show the following.

Proposition 3.5. Let $\varepsilon \in(0,1]$ and $d \in \mathbb{N}$ be fixed. Then, for each point $\mathbf{x} \in \Delta_{1}$, there is a non-negative integer $\ell_{\mathbf{x}} \in \mathbb{N}$ and an open neighbourhood $W_{\mathbf{x}}$ of $\mathbf{x}$ in $\mathbb{R}^{d}$, such that if $\mathbf{p} \in \Omega \cap W_{\mathbf{x}}$ is $\varepsilon$-hollow then its smallest weight $n_{\min }(\mathbf{p})$ satisfies $n_{\min }(\mathbf{p}) \leq \ell_{\mathbf{x}}$.

To prove this we introduce the following notation. For each set $U$ with $\mathbf{x} \in U \subseteq \mathbb{R}^{d}$ we define $\Delta_{U, \varepsilon}=\bigcap_{\mathbf{q} \in U} \Delta_{\mathbf{q}, \varepsilon}$, and we let $\mathcal{G}_{U, \varepsilon}$ be the family of all subgroups of $\mathbb{R}^{d}$ containing $\mathbb{Z}^{d}$ and not meeting $\Delta_{U, \varepsilon}^{\circ}$. Observe that

$$
U \supseteq U^{\prime} \quad \Rightarrow \quad \Delta_{U, \varepsilon} \subseteq \Delta_{U^{\prime}, \varepsilon} \quad \Rightarrow \quad \mathcal{G}_{U, \varepsilon} \supseteq \mathcal{G}_{U^{\prime}, \varepsilon} .
$$

We are interested in the case where $U$ is a neighbourhood of $\mathbf{x}$. 
Lemma 3.6. Let $B_{1} \supset B_{2} \supset \ldots$ be a countable base of neighbourhoods of $\mathbf{x}$, so that $\bigcap_{r \in \mathbb{N}} B_{r}=\{\mathbf{x}\}$. Then $\bigcup_{r \in \mathbb{N}} \Delta_{B_{r}, \varepsilon}^{\circ}=\Delta_{\mathbf{x}, \varepsilon}^{\circ}$.

Proof. The inclusion $\bigcup_{r \in \mathbb{N}} \Delta_{B_{r}, \varepsilon}^{\circ} \subseteq \Delta_{\mathbf{x}, \varepsilon}^{\circ}$ is immediate. For the other direction, if $\mathbf{y} \in \Delta_{\mathbf{x}, \varepsilon}^{\circ}$ then

$$
\begin{aligned}
\mathbf{x} \in\left\{\mathbf{z} \mid \mathbf{y} \in \Delta_{\mathbf{z}, \varepsilon}^{\circ}\right\} & =\left\{\mathbf{z} \mid \exists \mathbf{w} \in \varepsilon \Delta^{\circ} \text { such that } \mathbf{y}=\mathbf{z}(1-\varepsilon)+\mathbf{w}\right\} \\
& =\left\{\mathbf{z} \mid \mathbf{y}-\mathbf{z}(1-\varepsilon) \in \varepsilon \Delta^{\circ}\right\}
\end{aligned}
$$

which is open because $\varepsilon \Delta^{\circ}$ is open and $\mathbf{z} \mapsto \mathbf{y}-\mathbf{z}(1-\varepsilon)$ is continuous.

Hence $\mathbf{y} \in \Delta_{\mathbf{z}, \varepsilon}^{\circ}$ for all $\mathbf{z}$ in some neighbourhood of $\mathbf{x}$, and in particular for all $\mathbf{z} \in B_{r}$ for some sufficiently large $r$. Hence $\mathbf{y} \in \bigcup_{r \in \mathbb{N}} \Delta_{B_{r}, \varepsilon}^{\circ}$.

By analogy with Definition 3.2 we say that a closed group $G$ with identity component $L$ is $\varepsilon$-hollow at $\mathbf{x}$ if $G \cap(\mathbf{x}+L) \cap \Delta_{\mathbf{x}, \varepsilon}^{\circ}=\varnothing$.

Observe that this includes all closed groups with $\mathbf{x} \notin G$, since in this case $G \cap(\mathbf{x}+L)$ is already empty. Our next two lemmas prepare the proof of Proposition 3.5, dealing separately with groups that are and are not $\varepsilon$-hollow at $\mathbf{x}$.

Lemma 3.7. Every $\mathbf{x} \in \Delta_{1}$ has an open neighbourhood $U_{\mathbf{x}}$ such that every closed group in $\mathcal{G}_{U_{\mathbf{x}}, \varepsilon}$ is $\varepsilon$-hollow at $\mathbf{x}$.

Proof. Let $B_{1} \supset B_{2} \supset \ldots$ be a countable base of neighbourhoods of $\mathbf{x}$. We will prove the following, which has Lemma 3.7 as the case $k=0$ :

For every $k \in\{0, \ldots, d\}$ there is an $r$ such that every closed group of dimension $\geq k$ in $\mathcal{G}_{B_{r}, \varepsilon}$ is $\varepsilon$-hollow at $\mathbf{x}$.

The proof of this is by induction on $d-k$. The base case $k=d$ is trivial since the only group of dimension $d$ is the whole space $\mathbb{R}^{d}$, and this group does not lie in $\mathcal{G}_{B_{1}, \varepsilon}$. (We assume that $\Delta_{B_{1}, \varepsilon}$ has non-empty interior: Lemma 3.6 allows us to do this.)

Now, for a fixed $k$, our induction hypothesis is that there is an $r$ such that every closed group of dimension greater than $k$ in $\mathcal{G}_{B_{r}, \varepsilon}$ is $\varepsilon$-hollow at $\mathbf{x}$. That is, every closed group in $\mathcal{G}_{B_{r}, \varepsilon}$ that is not $\varepsilon$-hollow at $\mathbf{x}$ has dimension at most $k$. By Theorem 3.1 $\mathcal{G}_{B_{r}, \varepsilon}$ contains finitely many maximal groups, all closed. Let us denote $G_{1}, \ldots G_{t}$ the ones of dimension $k$ that are not $\varepsilon$-hollow (if any), and let $L_{1}, \ldots, L_{t}$ be their corresponding identity components. Observe that, although $\mathcal{G}_{B_{r}, \varepsilon}$ may contain additional non- $\varepsilon$ hollow groups of dimension $k$, apart from the $G_{i}$ 's, any such group must be contained in one of the $G_{i}$ 's and, in particular, its identity component must equal the corresponding $L_{i}$.

For each $i \in\{1, \ldots, t\}$, since $G_{i}$ is non- $\varepsilon$-hollow, $\mathbf{x}+L_{i}$ meets $\Delta_{\mathbf{x}, \varepsilon}^{\circ}$ by Lemma 3.6, $\mathbf{x}+L_{i}$ meets $\Delta_{B_{r_{i}}, \varepsilon}^{\circ}$ for some $r_{i}$. In particular, $\mathcal{G}_{B_{r_{i}}, \varepsilon}$ contains 
neither $G_{i}$ nor any other group whose identity component equals $L_{i}$. Obviously, the same holds for any $r \geq r_{i}$.

Hence, taking $r^{\prime}=\max \left\{r_{1}, \ldots, r_{t}\right\}$ we have that $\mathcal{G}_{B_{r^{\prime}}, \varepsilon}$ does not contain any group with identity component equal to any of the $L_{i}$ 's. Since $B_{r^{\prime}} \supset B_{r}$ we have $\mathcal{G}_{B_{r^{\prime}}, \varepsilon} \subset \mathcal{G}_{B_{r}, \varepsilon}$, and hence all the non- $\varepsilon$-hollow groups in $\mathcal{G}_{B_{r^{\prime}}, \varepsilon}$ are non- $\varepsilon$-hollow groups in $\mathcal{G}_{B_{r}, \varepsilon}$ too, but necessarily of smaller dimension.

Lemma 3.8. Let $\mathbf{x} \in \Delta_{1}$ and let $G$ be a closed group containing $\mathbb{Z}^{d}$ and $\varepsilon$-hollow at $\mathbf{x}$. Then there is a neighbourhood $W_{G}$ of $\mathbf{x}$ and a natural number $\ell_{G}$ such that every $\mathbf{p} \in \Omega \cap G \cap W_{G}$ has $n_{\min }(\mathbf{p}) \leq \ell_{G}$.

Proof. Let $L$ be the identity component of $G$. There are three possibilities:

- If $\mathbf{x} \notin G$, simply take $W_{G}=\mathbb{R}^{d} \backslash G$ and $\ell_{G}=0$.

- If $L \subset H_{0}$, then $\pi_{H_{0}}(G)=G /\left(G \cap H_{0}\right) \subset \mathbb{R}$ is discrete. Let $s$ be its minimum in $(1, \infty)$. We can take $W_{G}=\left\{\mathbf{p} \mid \Sigma p_{i}<s\right\}$ and $\ell_{G}=0$, since $\Omega \cap G \cap W_{G}=\varnothing$.

- If $\mathbf{x} \in G$ and $L \not \subset H_{0}$, then $\mathbf{x}+L \subset G$ but $(\mathbf{x}+L) \cap \Delta_{\mathbf{x}, \varepsilon}^{\circ}=\varnothing$, because $G$ is $\varepsilon$-hollow. But then $L+\mathbf{x}$ does not meet $\Delta_{1}^{\circ}$, so we may apply Proposition 3.3 to $L$. We then get an $\ell_{G}$ such that for every $\mathbf{p} \in \Omega \cap(\mathbf{x}+L)$ we have that the minimum weight of $\mathbf{p}$ is bounded by $\ell_{L}$. We can then take $W_{G}=\mathbb{R}^{d} \backslash(G \backslash(\mathbf{x}+L))$, so that $G \cap W_{G}=\mathbf{x}+L$ and $\Omega \cap G \cap W_{G}=\Omega \cap(\mathbf{x}+L)$.

We can now prove Proposition 3.5.

Proof of Proposition 3.5. By Lemma 3.7, $\mathbf{x}$ has an open neighbourhood $U_{\mathbf{x}}$ such that every group in $\mathcal{G}_{U_{\mathbf{x}}, \varepsilon}$ that contains $\mathbf{x}$ is $\varepsilon$-hollow. By Theorem 3.1 , $\mathcal{G}_{U_{\mathbf{x}}, \varepsilon}$ has a finite number of maximal elements, all closed and $\varepsilon$-hollow at $\mathbf{x}$, which we denote $G_{1}, \ldots, G_{t}$. By Lemma 3.8, each $G_{i}$ gives a neighbourhood $W_{i}$ of $\mathbf{x}$ and a natural number $\ell_{i}$ such that every $\mathbf{p} \in \Omega \cap G_{i} \cap W_{i}$ has $n_{\min }(\mathbf{p}) \leq \ell_{i}$.

Now it is enough to take $W_{\mathbf{x}}=U_{\mathbf{x}} \cap\left(\bigcap_{i} W_{i}\right)$ and $\ell_{\mathbf{x}}=\max \ell_{i}$. Indeed, let $\mathbf{p} \in W_{\mathbf{x}} \cap \Omega$ be $\varepsilon$-hollow, so that $\Delta_{\mathbf{p}, \varepsilon} \cap \Lambda_{\mathbf{p}}=\varnothing$. Since $\mathbf{p} \in W_{\mathbf{x}}$, we have $\Delta_{\mathbf{p}, \varepsilon} \supset \Delta_{W_{\mathbf{x}}, \varepsilon} \supset \Delta_{U_{\mathbf{x}}, \varepsilon}$. In particular, the group $\Lambda_{\mathbf{p}}$ is in $\mathcal{G}_{U_{\mathbf{x}}, \varepsilon}$, and hence is contained in one of the $G_{i}$ 's. Thus $\mathbf{p} \in \Omega \cap G_{i} \cap W_{i}$.

\subsection{The general case of Birkar's conjecture}

We are now in a position to give the proof of Theorem 1.3, settling Conjecture 1.2 completely.

Proof of Theorem [1.3. Fix $\varepsilon \in(0,1]$. For each $\mathbf{x} \in \Delta_{1}$, choose $\ell_{\mathbf{x}}$ and $W_{\mathbf{x}}$ as in Proposition 3.5, with $\ell_{\mathbf{x}}$ as small as possible. For a non-negative integer $\ell$, define $\Delta_{1}(\ell):=\left\{\mathbf{x} \in \Delta_{1} \mid \ell_{\mathbf{x}} \leq \ell\right\}$. Then $\Delta_{1}(\ell)$ is relatively open in $\Delta_{1}$, 
because if $\mathbf{y} \in W_{\mathbf{x}} \cap \Delta_{1}$ then $\ell_{\mathbf{y}}<\ell_{\mathbf{x}}$. Moreover, the $\left(\Delta_{1}(\ell)\right)_{\ell \in \mathbb{N}}$ obviously form an increasing sequence and they cover $\Delta_{1}$. Observe, for example, that $\Delta_{1}^{\circ} \subseteq \Delta_{1}(0)$, because if $\mathrm{x} \in \Delta_{1}^{\circ}$ and $G \cap(\mathrm{x}+L)$ meets $\Delta_{1}^{\circ}$ then $L \subset H_{0}$. Put differently, Proposition 3.3 is not needed on $\Delta_{1}^{\circ}$.

By compactness, there is an open subset $W=\bigcup_{\mathbf{x} \in \Delta_{1}^{\circ}} W_{\mathbf{x}}$ and an integer $\ell_{W}$ such that $\Delta_{1} \subset W$ and every $\varepsilon$-hollow $\mathbf{p} \in \Omega \cap W$ has $n_{\min }(\mathbf{p}) \leq \ell_{W}$. On the other hand, if $\mathbf{p} \in 2 \Omega$ then $V<1$, and since $\Omega \backslash(2 \Omega \cup W)$ is compact, the index (hence the minimum weight) of all $\mathbf{p} \in \Omega \backslash U$ has a global upper bound.

\section{Terminal and canonical bounds}

Throughout this section we take $\varepsilon=1$, so that we are considering only canonical and terminal singularities. In these cases we compute more explicit bounds, assuming that $\operatorname{dim} L$ or $\operatorname{codim} L$ is small. Combining these bounds with the classification of empty 4-simplices in [9] we give precise bounds in the terminal 4-fold case: that is, a precise answer to Question 1.1.

\subsection{Bounds in terms of width}

We first rework the bound of Proposition 3.3 in terms of the lattice width of $\operatorname{Conv}(S)=\pi_{L}(\Delta)$.

Definition 4.1. A linear functional $f: \mathbb{R}^{d} \rightarrow \mathbb{R}$ is called primitive with respect to a lattice $\Lambda$ if $f(\Lambda)=\mathbb{Z}$.

The width of a lattice polytope $\Pi$ in the direction of $f$ is the length of the interval $f(\Pi)$. Its facet width with respect to a facet $F$ is the width in the direction of the unique (up to a sign) primitive linear functional that is constant on $F$.

Let $G \subseteq \mathbb{R}^{d}$ be a closed group containing $\mathbb{Z}^{d}$ and not meeting $\Delta^{\circ}$, with identity component $L$. We keep the notation from Subsection 3.2, and we let $\Lambda_{G}=\pi_{L}(G)$, which is a lattice in $\mathbb{R}^{d} / L$, and put

$$
\ell_{G}=\max \left\{n_{\min }(\mathbf{p}) \mid \mathbf{p} \in \Omega \cap G\right\},
$$

i.e. the best possible bound for the smallest weight in $G$.

Proposition 4.2. $\ell_{G}$ is bounded by the maximum facet width of $\pi_{L}(\Delta)$ with respect to $\Lambda_{G}$.

Proof. Suppose first that $L \not \subset H_{0}$ and let $H$ be a facet-supporting hyperplane of $\pi_{L}(\Delta)=\operatorname{Conv}(S)$. We normalise the distance to $H$ by taking $f$ to be the primitive linear functional constant on $H$ and $\operatorname{dist}(H, \mathbf{x})=$ $|f(\mathbf{x})-f(H)|$. Then $1 \leq \operatorname{dist}\left(H, \mathbf{s}_{i}\right) \in \mathbb{N}$ for every $\mathbf{s}_{i} \notin H$ and $\operatorname{dist}(H, 0)$ is 
bounded above by the facet width with respect to the facet contained in $H$. Hence the statement follows from Proposition 3.3.

If $L \subset H_{0}$ then $\pi_{L}\left(H_{1}\right)$ is a facet-supporting hyperplane of $\pi_{L}(\Delta)$. If $\mathbf{p} \in \Omega \cap G$ then $\pi_{L}(\mathbf{p}) \in \Lambda_{G}$ and is strictly separated from $\pi_{L}(\Delta)$ by $\pi_{L}\left(H_{1}\right)$. So if $f$ is the primitive linear functional constant on $\pi_{L}\left(H_{1}\right)$, then $f_{1}:=$ $f\left(\pi_{L}\left(H_{1}\right)\right)$ is the facet width of $\pi_{L}(\Delta)$ with respect to $\pi_{L}\left(H_{1}\right)$, and $f(\mathbf{p}) \geq$ $f_{1}+1$. Hence $\Sigma p_{i} \geq \frac{f_{1}+1}{f_{1}}$, so $V \leq f_{1}$ and therefore $n_{\min }(\mathbf{p}) \leq f_{1}$.

Corollary 4.3. With the notation of Proposition 4.2.

(a) If $\pi_{L}(\Delta)$ has width equal to 1 in some lattice direction then $\ell_{G} \in\{0,1\}$. This is always the case if $\operatorname{dim} L=d-1$.

(b) If $\operatorname{dim} L=d-2$, then $\ell_{G} \in\{0,1,2\}$.

Proof. (a) Let $f$ be a primitive functional giving width 1 to $\Delta / L$, and $\widetilde{f}$ its pull-back to $\mathbb{R}^{d}$. Then $G^{\prime}:=G+\operatorname{Ker}(\widetilde{f})$ is a closed group containing $G$ and not intersecting $\Delta^{\circ}$, which implies $\ell_{G} \leq \ell_{G^{\prime}}$.

Thus there is no loss of generality in assuming $\operatorname{dim} L=d-1$. In this case $L=\operatorname{Ker}(\widetilde{f})$, so $\pi_{L}(\Delta)=f(\Delta)$ is a hollow lattice polytope of dimension 1 , that is, a unit segment. This has facet width 1 with respect to every facet, so Proposition 4.2 gives the statement.

(b) Here $\pi_{L}(\Delta)$ is a hollow lattice polytope of dimension 2. This implies $\pi_{L}(\Delta)$ either has width 1 or equals (modulo an affine isomorphism of the lattice) the triangle $\operatorname{Conv}((0,0),(2,0),(0,2))$ (see, e.g., [8]). This triangle has width 2 with respect its to all its three facets.

We can now recover Kawakita's result on the terminal weighted blowups in dimension 3.

Corollary 4.4 ([10, Theorem 3.5]). The weighted blowup $\mathbb{A}_{\mathbf{n}}^{3}$ has terminal singularities if and only if the weights are $(1, a, b)$, with $a$ and $b$ coprime.

Proof. This follows immediately from Corollary 4.3(a) and the theorem of White [16] that all empty 3 -simplices have width 1.

\subsection{Groups of dimension 1}

For our application to $d=4$ in Subsection 4.3 below, we want to consider the case $\operatorname{dim} L=1$ more carefully. In this case let $\left(a_{1}, \ldots, a_{d}\right) \in \mathbb{Z}^{d}$ be a primitive integer vector in $L$, which is unique up to sign, and let $a_{0}:=$ $\sum_{i=1}^{d} a_{i}$. The vector $\mathbf{a}:=\left(a_{0}, \ldots, a_{d}\right) \in \mathbb{Z}^{d+1}$ is called the $(d+1)$-tuple of $L$. We assume $L \nsubseteq H_{0}$, which is equivalent to $a_{0} \neq 0$.

Lemma 4.5. Suppose $\mathbf{p} \in \Omega$ and that $\operatorname{dim} L=1$, and that $(\mathbf{p}+L) \cap \Delta^{\circ}=\varnothing$. Then $n_{\min }(\mathbf{p}) \leq \max _{i=1, \ldots, d}\left\{-a_{i} / a_{0}\right\}$. 
Proof. The set $S=\left\{0, \mathbf{s}_{1}, \ldots, \mathbf{s}_{d}\right\}$ affinely spans $\mathbb{R}^{d} / L \cong \mathbb{R}^{d-1}$ and has $d+1$ points, so it has a unique (modulo a scalar factor) affine dependence. Since $\sum_{i=1}^{d} a_{i} \mathbf{e}_{i} \in L$, the coefficient vector of that dependence is precisely $\mathbf{a}$.

To bound the minimum weight we use Proposition 3.3 . Let $H$ be a facetsupporting hyperplane of $\operatorname{Conv}(S)$. If $0 \in H$ then $\ell_{H}=0$ in Proposition 3.3 , If $0 \notin H$ then, since $L \not \subset H_{0}$, there must be an $i$ with $\mathbf{s}_{i} \notin H$. Thus $H$ contains all of $S$ except for 0 and a single $\mathbf{s}_{i}$. Applying the affine dependence a to the affine functional vanishing on $H$ gives $\operatorname{dist}(H, 0) a_{0}+\operatorname{dist}\left(H, \mathbf{s}_{i}\right) a_{i}=$ 0 , which finishes the proof since

$$
\min _{\mathbf{s}_{j} \notin H} \frac{\operatorname{dist}(H, 0)}{\operatorname{dist}\left(H, \mathbf{s}_{j}\right)}=\frac{\operatorname{dist}(H, 0)}{\operatorname{dist}\left(H, \mathbf{s}_{i}\right)}=-\frac{a_{i}}{a_{0}} .
$$

We also have the following alternative bound, which is better than the previous one in a few critical cases.

Lemma 4.6. Let $\mathbf{p} \in \Omega$ be such that $\mathbf{n}=V \mathbf{p} \in \mathbb{N}^{d}$, where $V=\frac{1}{-1+\Sigma p_{i}}$ as usual. Suppose that there is a proper subset $J \subset\{1, \ldots, d\}$ such that

$$
\sum_{i \in J} p_{i}-s \sum_{i=1}^{d} p_{i} \in \mathbb{Z}
$$

for a positive integer $s$. Then either $\sum_{i \in J} n_{j} \leq s$ or else $n_{i}=0$ for all $i \notin J$.

Proof. Multiplying the equation in the statement by $V$ we obtain that

$$
\sum_{i \in J} n_{i}-s(V+1) \in V \mathbb{Z}
$$

so $\sum_{i \in J} n_{i} \equiv s(\bmod V)$. Since $\Sigma n_{i}=V+1$, either $n_{i}=0$ for every $i \notin J$, or $\sum_{i \in J} n_{i} \leq V$. The latter, together with $\sum_{i \in J} n_{i} \equiv s(\bmod V)$, implies $\sum_{i \in J} n_{i} \leq s$.

\subsection{Terminal 4-fold case}

Now we consider the case $d=4$, where there is an extensive history. Notice that another interpretation of Corollary 2.6 is that $\mathbb{A}_{\mathbf{n}}^{d}$ has terminal (or canonical) singularities if and only if the cyclic quotient singularity $\frac{1}{V} \mathbf{n}$ is terminal (or canonical), where $V=-1+\Sigma n_{i}$.

In fact any non-Gorenstein terminal quotient singularity in dimension 4 is cyclic, but this fails in higher dimension: see [2] for both of these facts. The singularity $\frac{1}{V} \mathbf{n}$ is never Gorenstein, but we note for completeness that Gorenstein cyclic terminal 4-fold singularities were classified in [13, and Gorenstein non-cyclic terminal 4-fold singularities in [1].

In dimension 4, a classification of non-Gorenstein terminal quotient singularities was begun experimentally in [12]. The first definite result was 
proved in [14] (another proof of the same result may be found in [5]): together with the results of [6] and [2], it implies that the list in [12] of such singularities of prime index is complete with possibly finitely many exceptions. Note, however, that the claim made in [2] that the results of [14] and [5] are valid for composite index is incorrect, as was pointed out in [4].

The complete classification of non-Gorenstein terminal quotient singularities in dimension 4 was recently given in [9], and we use it to prove Theorem 1.4.

In [9, Section 2] hollow simplices are divided into fine families. Two hollow lattice simplices $\Delta_{1}$ and $\Delta_{2}$ in $\mathbb{R}^{d}$, with $\operatorname{Vx}\left(\Delta_{i}\right)=\left\{\mathbf{v}_{i j}\right\} \subset \mathbb{Z}^{d}$, lie in the same fine family if there is an integer $k \leq d$ and integer affine maps $\pi_{i}: \mathbb{Z}^{d} \rightarrow \mathbb{Z}^{k}$ such that $\pi_{1}\left(\operatorname{Vx}\left(\Delta_{1}\right)\right)=\pi_{2}\left(\operatorname{Vx}\left(\Delta_{2}\right)\right)=S$ and $\operatorname{Conv}(S)$ is hollow. Here $S=\left\{\mathbf{s}_{0}, \ldots, \mathbf{s}_{d}\right\}$ is to be thought of as a multiset: that is, there is a permutation $\sigma$ of $\{0, \ldots, d\}$ such that $\pi_{1}\left(\mathbf{v}_{1 \sigma(j)}\right)=\pi_{2}\left(\mathbf{v}_{2 j}\right)$ for all $j$.

As before, if $G$ is a closed group containing $\mathbb{Z}^{d}$ and with $G \cap \Delta^{\circ}=\varnothing$ then $\pi_{L}(\Delta)$ is a hollow lattice polytope with respect to the lattice $\Lambda_{G}=\pi_{L}(G)$. Thus the rational points in $G$ parametrise (perhaps part of) a fine family of hollow simplices: each point $\mathbf{p} \in G \cap \mathbb{Q}^{d}$ corresponds, as in Corollary 2.6, to the standard simplex $\Delta \subset \mathbb{R}^{d}$ considered with respect to $\Lambda_{\mathbf{p}}$. In this situation we say $\mathbf{p}$ is a generating point of that hollow simplex. This relation makes Theorem 3.1 equivalent to [9, Corollary 2.7].

The case $L=\{0\}$ corresponds to the sporadic hollow simplices that do not project to hollow polytopes of lower dimension: more generally, the codimension of $L$, which we have called $k$ here, is the same as the parameter $k$ in [9, Theorem 1.6]. In particular, cases $k=1,2,3,4$ of [9, Theorem 1.6] correspond exactly to the cases $\operatorname{dim} L=3,2,1,0$ in our setting. We prove Theorem 1.4 separately for each value of $k$. We have already done $k=1$ and $k=2$.

Proposition 4.7. If a blowup $\mathbb{A}_{\mathbf{n}}^{4}$ of $\mathbb{A}^{4}$ belongs to the case $k=1$ then $n_{\min } \leq 1$, and if $k=2$ then $n_{\min } \leq 2$.

Proof. These are just parts (a) and (b) of Corollary 4.3 .

For the case $k=3$, the most interesting one, we analyse the bounds from Subsection 4.2. The index of a family parametrised by a group $G$ as above is defined to be the index $\left|G: L+\mathbb{Z}^{d}\right|$. A family is called primitive if its index is 1 , and non-primitive otherwise.

The classification in [9] for $k=3$ consists of two lists: one of 29 primitive quintuples Q1-Q29 (the same as the list of quintuples that appears in [12]), and one of 17 non-primitive quintuples N1-N17.

A primitive family is fully determined by $L$. In the case $\operatorname{dim} L=1$ and $d=4$ we specify $L$ via a quintuple $\mathbf{q}=\left(q_{1}, \ldots, q_{5}\right)$ with $\sum q_{i}=0$, defined by the property that $\mathbb{R} \mathbf{q}$ parametrises $\left(L+\mathbb{Z}^{4}\right) / \mathbb{Z}^{4}$ in barycentric coordinates 
with respect to the standard simplex. As shown in [9], the quintuple q can also be interpreted as the affine dependence among the points in $S=$ $\pi_{L}\left(\left\{0, \mathbf{e}_{1}, \ldots, \mathbf{e}_{4}\right\}\right)$. Thus, modulo a permutation of the entries, $\mathbf{q}$ is the same as the vector $\mathbf{a}=\left(a_{0}, \ldots, a_{4}\right)$ that we used in Lemma 4.5. However, in order to apply Lemma 4.5 we need to specify which of the entries $q_{l}$ will be considered the distinguished entry $a_{0}$.

A more concrete interpretation of the quintuple is as follows: for each $V \in \mathbb{N}$, the family corresponding to $\mathbf{q}$ contains a unique (modulo affineinteger isomorphism) hollow simplex of index $V$; the generating point $\mathbf{p}$ of this simplex can be chosen to be $\mathbf{p}=\frac{1}{V}\left(a_{1}, \ldots, a_{d}\right)$, where $\left(a_{1}, \ldots, a_{d}\right)$ is obtained from $\mathbf{q}$ by deleting the entry $q_{l}=a_{0}$ corresponding to the origin and permuting the rest. The generating point is only important modulo $\mathbb{Z}^{4}$.

In the non-primitive case a family is determined by not only $L$ or $\mathbf{q}$, but also by information on the group $G /\left(L+\mathbb{Z}^{4}\right)$. In $[9$ and in the table below this is expressed by adding to $\mathbf{q}$ a vector of the form $V \mathbf{r}$ (or of the form $\pm V \mathbf{r}$, for the non-primitive quintuples of index greater than 2, which are N7-N17). Observe, however, that the statement of Lemma 4.5 depends only on $L$, so only the q part plays any role in it. The part $V \mathbf{r}$ is only relevant when we apply Lemma 4.6. Since we will do this only for one non-primitive case, namely N5, we defer the details on how to interpret $V \mathbf{r}$ to when we need it.

We now list the quintuples, with the conventional labels Q1-Q29 and N1-N17.

\begin{tabular}{l|c|c|c||c|c} 
Case & Quintuple & Case & Quintuple & Case & Quintuple \\
\hline & & & & & \\
Q1 & $9,1,-2,-3,-5$ & Q18 & $15,1,-3,-5,-8$ & N1 & $6+\frac{V}{2}, 1,-2,-2+\frac{V}{2},-3$ \\
Q2 & $9,2,-1,-4,-6$ & Q19 & $15,2,-1,-6,-10$ & N2 & $4,3,-1,-2+\frac{V}{2},-4+\frac{V}{2}$ \\
Q3 & $12,3,-4,-5,-6$ & Q20 & $15,4,-2,-5,-12$ & N3 & $8,1,-2+\frac{V}{2},-3,-4+\frac{V}{2}$ \\
Q4 & $12,2,-3,-4,-7$ & Q21 & $18,1,-4,-6,-9$ & N4 & $6+\frac{V}{2}, 3,-1,-2+\frac{V}{2},-6$ \\
Q5 & $9,4,-2,-3,-8$ & Q22 & $18,2,-5,-6,-9$ & N5 & $8,3,-1,-4+\frac{V}{2},-6+\frac{V}{2}$ \\
Q6 & $12,1,-2,-3,-8$ & Q23 & $18,4,-1,-9,-12$ & N6 & $12,1,-3,-4+\frac{V}{2},-6+\frac{V}{2}$ \\
Q7 & $12,3,-1,-6,-8$ & Q24 & $20,1,-4,-7,-10$ & N7 & $3,1,-1 \pm \frac{V}{3},-1 \pm \frac{2 V}{3},-2$ \\
Q8 & $15,4,-5,-6,-8$ & Q25 & $20,1,-3,-8,-10$ & N8 & $3,2,-1,-1 \pm \frac{2 V}{3},-3 \pm \frac{V}{3}$ \\
Q9 & $12,2,-1,-4,-9$ & Q26 & $20,3,-4,-9,-10$ & N9 & $3,2,-1,-2 \pm \frac{V}{3},-2 \pm \frac{2 V}{3}$ \\
Q10 & $10,6,-2,-5,-9$ & Q27 & $20,3,-1,-10,-12$ & N10 & $4 \pm \frac{V}{3}, 2,-1,-1 \pm \frac{2 V}{3},-4$ \\
Q11 & $15,1,-2,-5,-9$ & Q28 & $24,1,-5,-8,-12$ & N11 & $6,1,-2,-2 \pm \frac{2 V}{3},-3 \pm \frac{V}{3}$ \\
Q12 & $12,5,-3,-4,-10$ & Q29 & $30,1,-6,-10,-15$ & N12 & $6,1,-1 \pm \frac{2 V}{3},-2,-4 \pm \frac{V}{3}$ \\
Q13 & $15,2,-3,-4,-10$ & & & N13 & $4,3,-1 \pm \frac{2 V}{3},-2,-4 \pm \frac{V}{3}$ \\
Q14 & $12,1,-3,-4,-6$ & & & N14 & $6,3 \pm \frac{V}{3},-1,-2 \pm \frac{V}{3},-6 \pm \frac{V}{3}$ \\
Q15 & $14,1,-3,-5,-7$ & & & N15 & $3 \pm \frac{V}{4}, 2,-1,-1 \pm \frac{V}{4},-3 \pm \frac{V}{2}$ \\
Q16 & $14,3,-1,-7,-9$ & & & N16 & $6,1 \pm \frac{V}{4},-1,-3 \pm \frac{V}{4},-3 \pm \frac{V}{2}$ \\
Q17 & $15,7,-3,-5,-14$ & & & N17 & $3,1 \pm \frac{V}{6},-1,-1 \pm \frac{V}{6},-2 \pm \frac{2 V}{3}$
\end{tabular}


In every case the entries are arranged so that

$$
q_{1}>q_{2}>0>q_{3} \geq q_{4} \geq q_{5} .
$$

With this convention, we have $\max \left\{-a_{j} / a_{0}\right\} \leq-q_{1} / q_{3}$ if $a_{0} \in\left\{q_{1}, q_{2}\right\}$ and $\max \left\{-a_{j} / a_{0}\right\} \leq-q_{5} / q_{2}$ if $a_{0} \in\left\{q_{3}, q_{4}, q_{5}\right\}$. Thus Lemma 4.5 implies the following. Observe that in the hypotheses of this statement we can write $<7$ instead of $\leq 6$ since all weights are integers.

Lemma 4.8. If a quintuple $\mathbf{q}$ (primitive or not) written as above satisfies

$$
\max \left\{-q_{1} / q_{3},-q_{5} / q_{2}\right\}<7
$$

then every blowup coming from that quintuple has $n_{\max } \leq 6$.

With this, we are now ready to prove the main result in this section, which gives Theorem 1.4 for the families with $\operatorname{dim} L=1$, that is, $k=3$.

Proposition 4.9. If a blowup $\mathbb{A}_{\mathbf{n}}^{4}$ of $\mathbb{A}^{4}$ belongs to the case $k=3$ (equivalently, $\operatorname{dim} L=1$ ) then $n_{\min } \leq 6$.

Proof. The reader may easily check that the only cases where Lemma 4.8 is not sufficient to prove a bound of 6 are the ones shown (with the ratio $q_{1}:-q_{3}$ or $-q_{5}: q_{2}$ that we do get) in the table below. In all the other cases, including the ones marked "- " in the table, the ratios $q_{1}:-q_{3}$ and $-q_{5}: q_{2}$ are strictly less than 7 . In the non-primitive quintuples this check is especially easy, since none of them has $-q_{5}>6$ and the only ones with

\begin{tabular}{|c|c|c|c|c|c|}
\hline quintuple & $q_{1}:-q_{3}$ & $-q_{5}: q_{2}$ & quintuple & $q_{1}:-q_{3}$ & $-q_{5}: q_{2}$ \\
\hline Q2 & $9: 1$ & - & Q20 & $15: 2$ & - \\
\hline Q6 & - & $8: 1$ & Q21 & - & $9: 1$ \\
\hline Q7 & $12: 1$ & - & Q23 & $18: 1$ & - \\
\hline Q9 & $12: 1$ & - & Q24 & - & $10: 1$ \\
\hline Q11 & $15: 2$ & $9: 1$ & Q25 & - & $10: 1$ \\
\hline Q15 & - & $7: 1$ & Q27 & $20: 1$ & - \\
\hline Q16 & $14: 1$ & - & Q28 & - & $12: 1$ \\
\hline Q18 & - & $8: 1$ & Q29 & - & $15: 1$ \\
\hline Q19 & $15: 1$ & - & N5 & $8: 1$ & - \\
\hline
\end{tabular}
$q_{1}>6$ are N3, N5, and N6.

Even where the bound exceeds 7 , the ratios $-q_{5} / q_{1}$ and $-q_{1} / q_{4}$ (hence also $\left.-q_{1} / q_{5}\right)$ are less than 7 , which implies that for the cases with $l=1,4,5$ the bound of Lemma 4.5 is at most 6 in every quintuple. Thus the eighteen quintuples in the table correspond to nineteen pairs (quintuple, $l$ ) that need to be checked: one of $l=2$ or $l=3$ for each of the quintuples, except for the quintuple Q11 where we have to check both.

Sixteen of the nineteen cases are primitive quintuples in which $q_{2}=1$ (if $l=2$ ) or $q_{3}=-1$ (if $l=3$ ). This is fortunate since in these cases it is particularly simple to apply Lemma 4.6. Indeed: 
- If $a_{0}=q_{2}=1$ then we can use $s=-q_{3}$ in the lemma, by letting $J$ be just one coordinate, the one corresponding to $q_{3}$.

- If $a_{0}=q_{3}=-1$ then we can use $s=q_{2}$ in the lemma, by letting $J$ be just one coordinate, the one corresponding to $q_{2}$.

That is, in these sixteen cases we can use $-q_{3}$ and $q_{2}$ as bounds instead of the bigger $-q_{5}$ and $q_{1}$, respectively. The worst value obtained is 6, for Q29 with $l=2$.

For the last three remaining cases we also apply Lemma 4.6 as follows:

- For $\mathrm{Q} 11=(15,1,-2,-5,-9)$ with $a_{0}=q_{3}=-2$, our generating point is $\mathbf{p}=\frac{1}{V}(15,1,-5,-9)$. Taking $J$ to be the first and fourth coordinates and $s=3$ we have $\sum_{i \in J} p_{i}-s \sum_{i=1}^{4} p_{i}=\frac{1}{V}((15-9)-3 \cdot 2)=0$. Thus, Lemma 4.6 gives $n_{1}+n_{4} \leq 3$.

- For $\mathrm{Q} 20=(15,4,-2,-5,-12)$ with $a_{0}=q_{3}=-2$, our generating point is $\mathbf{p}=\frac{1}{V}(15,4,-5,-12)$. Taking $J$ to be the first and third coordinates and $s=5$ we have $\sum_{i \in J} p_{i}-s \sum_{i=1}^{d} p_{i}=\frac{1}{V}((15-5)-5 \cdot 2)=0$. Thus, Lemma 4.6 gives $n_{1}+n_{3} \leq 5$.

- For N5 the quintuple is expressed as $\left(8,3,-1,-4+\frac{V}{2},-6+\frac{V}{2}\right)$, that is, as $\mathbf{q}+V \mathbf{r}$ with $\mathbf{q}=(8,3,-1,-4,-6)$ and $\mathbf{r}=\frac{1}{2}(0,0,0,1,1)$. The interpretation of this is that hollow simplices in this family are those with generating point (in barycentric coordinates) equal to

$$
\frac{1}{V}(8,3,-1,-4,-6)+\frac{1}{2}(0,0,0,1,1) .
$$

See [9] for more details.

Since $l=3$, we have to omit the third coordinate and get

$$
\mathbf{p}=\frac{1}{V}\left(8,3,-4+\frac{V}{2},-6+\frac{V}{2}\right),
$$

whose sum of coordinates is equal to $1+\frac{1}{V}$.

Taking $J$ to be just the second coordinate and $s=3$ we have

$$
\sum_{i \in J} p_{i}-s \sum_{i=1}^{d} p_{i}=\frac{3}{V}-3\left(1+\frac{1}{V}\right)=-3 \in \mathbb{Z},
$$

so Lemma 4.6 gives $n_{2} \leq 2$.

Thus, in all cases we get a bound of at most 6 for the smallest weight. 
Remark 4.10. The bounds obtained by these methods are not sharp for each individual quintuple and choice of $l$, but the overall bound in Proposition 4.9 is sharp. For example, the blowup $\mathbb{A}_{(V-30,6,10,15)}^{4}$, arising from Q29 with $l=2$, has terminal singularities whenever $V$ is coprime with 30, and has minimum weight equal to 6 for every $V \geq 37$. This gives an infinite family of blowups of $\mathbb{A}^{4}$ with terminal singularities and $n_{\min }=6$.

To finish the proof of Theorem 1.4 we need to look at the case $k=4$, that is, at the 2641 sporadic terminal 4-simplices enumerated in [9. The full list is publicly available, and each simplex is expressed as a pair $(V, \mathbf{b})$ with $V \in \mathbb{N}$ and $\mathbf{b} \in\left(\mathbb{Z}_{V}\right)^{5}$ where, as before, $V$ equals the (normalised) volume and $\frac{1}{V} \mathbf{b}$ are the barycentric coordinates (modulo an integer vector, which does not affect the lattice) for a generator of $\Lambda / \mathbb{Z}^{d}$.

Each such simplex corresponds to five terminal quotient singularities (perhaps not distinct, if the simplex has symmetries) but not all such singularities correspond to blowups of $\mathbb{A}^{4}$. The conditions for that are that:

- the corresponding entry $b_{l}$ of $\mathbf{b}$ is coprime to $V$, so that by multiplying by a unit in $\mathbb{Z}_{V}$ we can assume that entry to be -1 , and

- after this multiplication, the representatives in $\{0, \ldots, V-1\}$ of the other four entries (remember that they are only important modulo $V$ ) add up to $V+1$.

When these conditions hold, the other four entries are the weights of a blowup of $\mathbb{A}^{4}$.

We have computationally checked the $2641 \times 5$ possibilities, obtaining the results summarised in the following statement.

Proposition 4.11. Among the $2641 \times 5$ sporadic terminal quotient singularities of dimension 4 there are 4620 blowups, all with $n_{\min } \leq 32$. The number $B$ of sporadic blowups with each possible value of $n_{\min }$ is as follows.

\begin{tabular}{c|c}
$n_{\min }$ & $B$ \\
\hline 1 & 0 \\
2 & 964 \\
3 & 804 \\
4 & 413 \\
5 & 468 \\
6 & 187 \\
7 & 408 \\
8 & 212
\end{tabular}

\begin{tabular}{c|c}
$n_{\min }$ & $B$ \\
\hline 9 & 194 \\
10 & 130 \\
11 & 178 \\
12 & 81 \\
13 & 137 \\
14 & 63 \\
15 & 63 \\
16 & 48
\end{tabular}

\begin{tabular}{c|c}
$n_{\min }$ & $B$ \\
\hline 17 & 65 \\
18 & 34 \\
19 & 57 \\
20 & 26 \\
21 & 16 \\
22 & 11 \\
23 & 23 \\
24 & 7
\end{tabular}

\begin{tabular}{c|c}
$n_{\min }$ & $B$ \\
\hline 25 & 12 \\
26 & 5 \\
27 & 5 \\
28 & 2 \\
29 & 3 \\
30 & 1 \\
31 & 2 \\
32 & 1
\end{tabular}

The unique blowup with $n_{\min }=32$ has $V=245$ and $\mathbf{n}=(32,41,71,102)$. The unique sporadic simplex of maximum volume $V=419$ produces two blowups with terminal singularities, with weight vectors

$$
(20,57,133,210) \quad \text { and } \quad(21,60,140,199) .
$$


Theorem 1.4 now simply summarises Propositions 4.7, 4.9 and 4.11

\section{References}

[1] R.E. Anno, Four-dimensional terminal Gorenstein quotient singularities, Mat. Zametki 73 (2003), 813-820 (Math. Notes 73 (2003), 769-776).

[2] M. Barile, D. Bernardi, A. Borisov \& J.-M. Kantor, On empty lattice simplices in dimension 4, Proc. Amer. Math. Soc. 139, no. 12, 4247-4253 (2011).

[3] C. Birkar, Anti-pluricanonical systems on Fano varieties, Ann. Math. 190 (2019), 345-463

[4] M. Blanco, C. Haase, J. Hofmann \& F. Santos, The finiteness threshold width of lattice polytopes, arXiv:1607.00798

[5] J.W. Bober, Factorial ratios, hypergeometric series, and a family of step functions, J. Lond. Math. Soc. 79 (2009), no. 2, 422-444.

[6] A. Borisov, On classification of toric singularities, J. Math. Sci. (New York) 94 (1999), 1111-1113.

[7] Y. Chen, On singularities of threefold weighted blowups, arXiv:1911.04726

[8] C.A.J. Hurkens, Blowing up convex sets in the plane, Linear Algebra Appl. 134 (1990), 121-128.

[9] O. Iglesias-Valiño \& F. Santos, The complete classification of empty lattice 4-simplices, arXiv:1908.08933

[10] M. Kawakita, Divisorial contractions in dimension three which contract divisors to smooth points, Invent. Math. 145 (2001), 105-119.

[11] J. Lawrence, Finite unions of closed subgroups of the $n$-dimensional torus, In: Applied Geometry and Discrete Mathematics, 433-441, DIMACS Ser. Discrete Math. Theoret. Comput. Sci. 4 Amer. Math. Soc., Providence, Rhode Island (1991).

[12] S. Mori, D. Morrison \& I. Morrison, On four-dimensional terminal quotient singularities, Math. Comp. 51 (1988), 769-786.

[13] D. Morrison \& G. Stevens, Terminal quotient singularities in dimensions three and four, Proc. Amer. Math. Soc. 90 (1984), 15-20.

[14] G.K. Sankaran, Stable quintuples and terminal quotient singularities, Math. Proc. Cambridge Philos. Soc. 107 (1990), 91-101.

[15] T. Oda, Convex Bodies and Algebraic Geometry, Ergebnisse der Mathematik und ihrer Grenzgebiete 15, Springer, Berlin 1988.

[16] G.K. White, Lattice tetrahedra, Canadian J. Math. 16 (1964), 389-396. 